\title{
ANALYSIS OF THE SEGMENTED FEATURES OF INDICATOR OF MINE PRESENCE
}

\author{
A. Krtalic \\ Faculty of Geodesy, University of Zagreb, Kaciceva 26, 10000 Zagreb, Croatia - kandrija@ gmail.com
}

\author{
Commission III, WG III/3
}

KEY WORDS: Indicator of mine presence, segmentation, general, geometric shapes, factor analysis

\begin{abstract}
:
The aim of this research is to investigate possibility for interactive semi-automatic interpretation of digital images in humanitarian demining for the purpose of detection and extraction of (strong) indicators of mine presence which can be seen on the images, according to the parameters of the general geometric shapes rather than radiometric characteristics. For that purpose, objects are created by segmentation. The segments represent the observed indicator and the objects that surround them (for analysis of the degree of discrimination of objects from the environment) in the best possible way. These indicators cover a certain characteristic surface. These areas are determined by segmenting the digital image. Sets of pixels that form such surface on images have specific geometric features. In this way, it is provided to analyze the features of the segments on the basis of the object, rather than the pixel level. Factor analysis of geometric parameters of this segments is performed in order to identify parameters that can be distinguished from the other parameters according to their geometric features. Factor analysis was carried out in two different ways, according to the characteristics of the general geometric shape and to the type of strong indicators of mine presence. The continuation of this research is the implementation of the automatic extraction of indicators of mine presence according results presented in this paper.
\end{abstract}

\section{INTRODUCTION}

The Republic of Croatia (RC) has been faced with the mine problems as one of the most difficult consequences of the war events in this area. From the very beginning of dealing with the demining problems, it has been essential to define the suspected hazardous area (SHA). During the years 2003 and 2004, HCR has performed the reconnaissance and marking of the entire area of RC, thus, $1174 \mathrm{~km}^{2}$ of the area was defined as mine suspected. At the end of 2015, the revision of the process started in order to maintain constantly updated data connected with SHA. The revisions are performed because SHA is defined in much larger area than really occupied. It has been done in order to reduce the risk of mine danger. The employees of the Croatian Mine Action Centre (HCR) have defined and redefined SHA according to the data from the Mine information System (MIS) (the data collected by inspecting the field, interviewing the population and the data from the military documents (mine records, military maps). RC has committed itself to solve the problem of landmines on its territory before 2019 year. In order to do so, it is necessary to collect new data about the depth of SHA along with classical demining and reduce SHA based on these data (without demining).

One of the methods is the collection of aerial images related to the depth of SHA and interpreting these images for the purpose of finding and extracting the indicators of mine presence and absence. The indicators of mine presence (IMP) (van Genderen, Maathuis, 1999), (ARC 2004), (Vanhuysse, 2002) are the objects within the SHA that is assumed to have been defended with mine-explosive obstacles (minefield). The indicators of mine absence (IMA), on the other hand, are the areas within the SHA that are considered free of mines. The Advanced Intelligence Decision Support System (AI DSS)
(Bajic et al., 2008) has been operatively used in the Republic of Croatia for capturing the additional information about the space deeper in the mine suspected area. The system was improved within the scope of the FP7 project Toolbox Implementation for Removal of Anti-Personnel Mines, Submunitions and UXO (TIRAMISU (URL3)) and has been offered to the humanitarian community to use it since 2015 . TIRAMISU Advanced Intelligence Decision Support System (T-AI DSS) is a tool to support the decision about the status of SHA (Krtalic and Bajic, 2013). T-AI DSS is a complex system that consists of three modules: Module for analytical assessment of data within MIS, Module for (aerial) data acquisition, Module for pre-processing and processing of data. Module for (aerial) data acquisition can be installed in various types of aircrafts. The system consists of sensors (cameras) that capture the data about the scene in visible, close infrared and thermal wave range (Bajić, 2010). The system applies scientific methods to analyse and process (image fusion is performed) all comparable available information, data and expert knowledge about the mine scene. There are also all available images outside the Module for (aerial) data acquisition (photogrammetric and satellite images) used. Thematic presentations of the impact and the interaction of the impacts of all IMPs (danger map) and IMA (proposed reduction map) (Krtalic, 2012) are made. Danger map is a thematic map presenting all IMPs within SHA with the action zones around them and with the level of estimated mine presence danger in these zones. Zones of influence are the distances from IMP where the mine-explosive obstacles are expected. They are determined by the experts in the field of humanitarian demining. Proposed reduction map is a thematic map presenting all IMAs within SHA. The experts in the domain of warfare and mining operations can make decisions about redefining of SHA more easily on the basis of the data captured from such thematic presentations. Danger maps are 
not the object of the interest in this paper, but it is important to emphasize the necessity of finding and extracting of IMPs and IMAs for their production.

Within the frame of the research carried out for this project, the material objects have been analysed at the territory assumed to have been defended by means of mine-explosive obstacles. Strong IMP (trench, battlement, bunker, shelter) are the objects the location of which can be definitely determined in the space and their environmental influence evaluated. The description of the strong IMP have been made in this paper by means of the features of general shapes of their segments.

The IMP occupy a certain characteristic area, and the sets of pixels creating them on the images have certain spectral and geometric features. Due to their dominant geometric features, they are divided into two groups: linear and surface indicators. Linearly strong indicators of mine presence (trench, battlement) are the surface objects whose length is essentially more dominant related to their width. The other indicators are surface indicators (shelter, bunker).

All strong IMP used in this analysis have been discovered by means of interactive interpretation of the mine scene:

on the basis of the images collected by means of plane multisensory systems for reconnaissance and supervision;

- $\quad$ by processing and interpretation of satellite images; - $\quad$ with orthophoto plans;

by analysing the field using digital terrain model.

\section{DEFINITION OF THE INDICATOR OF MINE PRESENCE}

Within the frame of the system of interactive extracting of indicators in the system intended to provide help in decisionmaking, there are also well known methods of processing the digital images used for the purpose of automatic detection and extracting of IMP (e.g. the usage of threshold, separation of characteristics and classification). The extracting of line objects is performed on the basis of spectral and geometric properties of indicators. It can be done on the basis of various tools for the processing of digital images provided by commercial software packages (e.g. the extracting of the object edges, i.e. of the jumps in the in pixel value intensity at the boundaries of an object) or the tools developed in some project (the tools from the project SMART (SMART, 2004) for monitoring certain line object (road trucker) making it possible for a user to select an initial pixel, and the tool shows on the screen all pixels with similar characteristics in an array (Acheroy and Yvinec, 2008)). These processing procedures depend on the objects in the scene, illumination, atmospheric conditions, and they are well known and used. However, more attention is paid to better defining of strong IMP (that the author has not found in so far published literature) using the properties of general shapes (it will be explained in more details further in the text).

In the analysis presented in this paper, the attention has been paid to better defining of strong IMP by means general shape features (asymmetry, border index, compactness, density, elliptic fit, length/width, radius of smallest enclosing ellipse, radius of largest enclosed ellipse, rectangular fit, roundness). The analysis is aiming to generate a subjective and objective key for the interpretation of strong IMP. A man (the human eye and contextual view on the scene) as an interpreter (of mine scene) can hardly be replaced by automatic methods of digital image processing. However, some methods of digital image processing (implemented in certain ways) may help the interpreter in the identification of certain anomalies in the scene and easier detection of indicators. The basic idea of interactive methods of semi-automatic (automatic by human) interpretation of digital images of SHA is to provide assistance to the interpreter in the interpretation of digital images rather than to replace him.

In order to analyse the indicators, they should first be found on the images and highlighted. It can be achieved in two ways. The first one is manual vectorising of an object when found by an interpreter on the image. It is a subjective method that depends on the skills of the interpreter. The other way is to find and extract the objects of interest after segmenting the digital image. In this way, the subjectivity of the interpreter is reduced and one focuses on the determining the parameters used for the performance of segmentation. For the purpose of the analysis in this article, the segmentation of images has been performed, where after the segments have been joined into objects that have presented the observed indicator and the surrounding objects in the best possible way. Thus created objects have been used to analyse the features at the object level, and not at the pixel level (the sets of pixels are analysed that form a certain object and thus represent this object better than individual pixels). The indicators have been analysed by means of certain general object features (Krtalic, 2011). The TAI DSS module for image acquisition for non-technical survey of the SHA consists of matrix cameras: Nikon D90, DuncanTech MS4100, Photon 320; and hyperspectral linear scanner (Imspector V9). Canon EOS 5D and MS3100 matrix cameras were used in previous versions of the module. The images used in the analysis are the result of remote sensing methods and are presented in the Table 1. Apart from the images acquired within T-AI DSS, there were also other images used that were acquired for various projects (ARC, SMART, TIRAMISU).

The analyses have been performed in the programme packages: for object classification Definiens Professional 5.0 (URL2) and for statistical processing STATISTICA (URL1).

\begin{tabular}{|l|c|r|c|}
\hline \multicolumn{1}{|c|}{ Sensor } & \multicolumn{1}{c|}{$\begin{array}{c}\text { Type of } \\
\text { Sensor/spectral }\end{array}$} & $\begin{array}{c}\text { No. of } \\
\text { channels }\end{array}$ & Source \\
\hline Canon EOS 5D & $\mathrm{CMOS}(0.4-0.7 \mu \mathrm{m})$ & 3 & [ITF 2008] \\
\hline Nikon D90 & $\mathrm{CSD}(0.4-0.7 \mu \mathrm{m})$ & 3 & [TTF 2010] \\
\hline MS-4100 camera & $3 \mathrm{CCD}(0.55-0.80)$ & 3 & {$[$ [TF 2010] } \\
\hline RMK camera & NIR $(0.40-0.90 \mu \mathrm{m})$ & 3 & [SMART] \\
\hline DAEDALUS & $0.42-14.0 \mu \mathrm{m}$ & 11 & [SMART] \\
\hline WorldView & $0.40-1.040 \mu \mathrm{m}$ & 1 & [ITF 2010] \\
\hline Ortophoto, & $0.4-0.7 \mu \mathrm{m}$ & 1 & [ITF 2008] \\
\hline
\end{tabular}

Table 1. Spectral characteristics of sensors and documents used in the analysis.

\subsection{Segmentation of digital images}

Segmentation of digital images (DEFINIENS, 2006) is the process of segmenting the image into smaller parts (objects) 
that have common properties (features, Figure 1). Typical techniques of the image segmentation are: a) joining the image segments based on some homogeneity measures, and b) separating the objects by means of emphasizing the edges using the gradients of numerical values (grey tone value level, digital value) between the neighbouring pixels (Navulur, 2006). For the purpose of this research, the segmentation has been carried out by means of the programme package Definiens Professional 5.0 (this software was available to the author, hence it was used for the segmentation), according to the heuristic model patented by Definiensa (Navulur, 2006), and it is based on five criteria:

scale parameter,

determination of homogeneity criteria by means of four parameters:

colour (grey tone value level),

shape,

smoothness,

compactness.

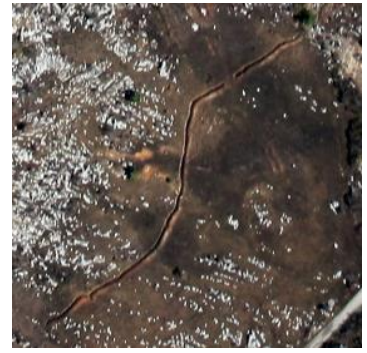

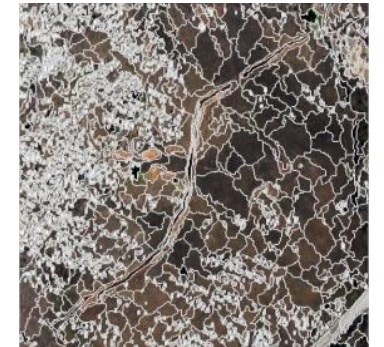

b
Figure 1. a) RGB image of the scene with a trench and shelter for heavy weapons, b) Segmented image on the basis of the following parameters: scale parameter: 25 , colour: 5 , shape: 5 , compactness; 3 , smoothness; 7 .

For the purpose of feature analysis of each indicator (object) on the digital image, it is necessary to determine the pixels that form the object in the image. Image segmentation is used for this procedure. After creating segments for radiometric and geometric analysis of the segments, statistical methods factor and cluster analysis were used. Related parameters as colour and shape, and smoothness and compactness. The sum of the coefficients of their impact is always 1 , and the condition is that the colour parameter can never be 0 . Segmentation is performed on a set of images of different resolutions and it is therefore possible to weigh the images according to the needs analysis and resolution of images. All these segmentation parameters depend on the scale of images (pixel size and resolution (ground sampling distance (GSD)) and the size of the object. Better GSD has resulted in better segmentation. However, since radiometric characteristics of the image are also applied for the creation of segments, all images of the analysed area have been used. Higher weight factor has been attributed to the images with better GSD. In this way, all radiometric characteristics of all images have been used (commercial matrix cameras with one CCD sensor: Canon EOS 5D, Nikon D90; multispectral matrix cameras with 3 CCD sensors: MS3100, MS4100). In the preparation of this work, the individual influence of each image on the final appearance of a segment has not been analysed. Subjective delineation (vectorization by hand) of indicators in digital images is replaced by segmentation based on similar features (Figure 2) that ultimately form a (strong) IMP or IMA. In this manner segmentation reduces subjectivity in extraction (delineating) of indicators in digital images.

The algorithm of multiresolution segmentation used in the analysis is a heuristically optimized procedure that locally minimizes the mean heterogeneity of image objects within the scope of image resolution (DEFINIENS, 2006). The scale parameter is an abstract term (value) determining maximally allowed heterogeneity of an object (segment) of image, while the homogeneity of an object is defined in the field for the determination of the homogeneity criteria (composition of homogeneity criterion). The scale parameter defines in fact the maximum standard deviation of the homogeneity criterion with regard to the weighted image levels for the resulting image objects, so that it gets bigger with the increased parameter value. The homogeneity criterion consists of four sub criteria that define the whole relative homogeneity of the resulting image object:

- shape defines texture homogeneity, and

- colour defines digital value (shades of gray) of the resulting object (they are connected with the relation: Colour $=1$-Shape) .

- smoothness is a parameter used to optimize smoothness,

- compactness is used to optimize the whole compactness of the resulting image object within the shape criteria. It should be pointed out that the smoothness and compactness are not opposite criteria, i.e. the object optimized by means of compactness can also be smoothed (DEFINIENS, 2006).

The segments (after segmentation) represent the observed indicator and the objects that surround them (for analysis of the degree of discrimination of objects from the environment, Figure 2, Figure 3, Figure 4, Figure 5) in the best possible way. Statistical analysis of radiometric and geometric parameters is performed in order to identify parameters that can be distinguished from the other parameters according to their features. If it cannot be done directly, with one processing, a combination of treatment is looked for that can extract the desired object (an indicator or some percentage of its surface) on the resulting image.

\subsection{Indicators in hilly-lowland terrain}

The spectral area in which the indicator features have been analysed at this territory is a visible part of EM spectrum (RGB, 0.4-0.7 $\mu \mathrm{m}$ ) within orthophoto or recorded with the sensor Canon EOS 5D. In this type of the terrain, the following has been analysed spectrally and radiometrically: trenches, battlements and buried artillery cover (or shelter for tank).

\subsubsection{Trench, battlement and buried artillery cover} Trench, battlement and various buried artillery covers appear mostly together on the scene, they are made of the same or similar material (natural material from the surrounding area Figure 2), hence, it is expected that their features are also similar. It is also supported by the fact that we deal here with some kind of "scene archaeology" (the term introduced by Bajic, M.), i.e. there is an attempt to discriminate the objects in the land terrain made 24 to 20 years ago and have not been used for at least twenty years.

There have been several segmentations carried out with various parameters. The segmentation: 25 5-5-3-7 has proved to be best for the creation of trench objects 2 and battlements 2 
from the Fig. 5-10b, and for the shelter 2 the segmentation: 5_5-5-3-7 (Figure 3). The formed segments describe the observed objects in the best way. The trench 3 consists of three objects with one of them characterizing the middle part in the best way, and the shelter 2 is presented with one image object (Figure 3b).

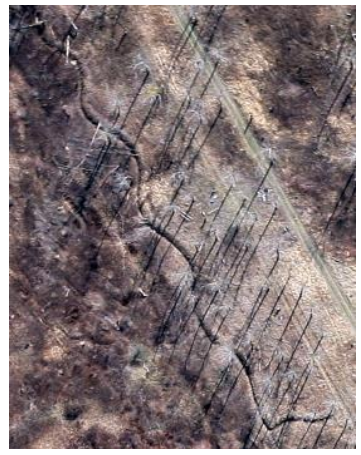

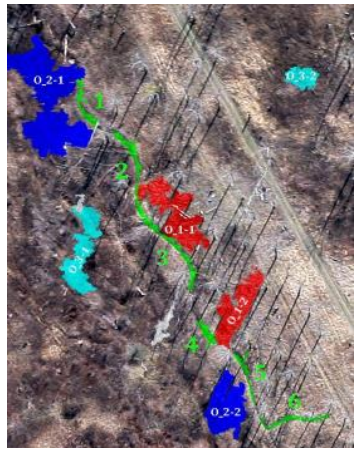

b
Figure 2. a) The cut part of the colour (RGB) image (Canon EOS 5D) of the trench 1 in the lowland type of the terrain, and b) the presentation of the trench (light green) sections, and of the surrounding patterns (red, dark blue, light blue) on the same image (ITF, 2008).

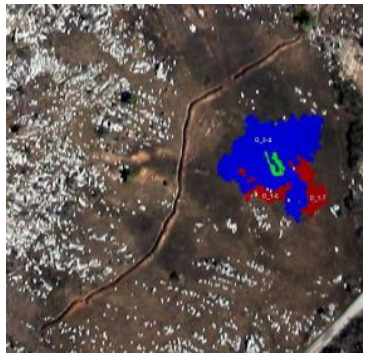

a

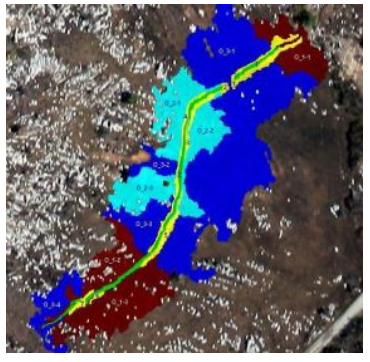

$\mathrm{b}$
Figure 3. a) The cut part of the colour (RGB) image (Canon EOS 5D) of the trench 3 and the buried artillery cover 2 (green) and the surrounding area (red and blue) in the hilly type of the terrain, and b) the presentation of the trench 3 (light green), the battlement 2 (yellow) and the surrounding pattern (red, light and dark blue) on the same image (ITF, 2008).

\subsection{Indicators in the hilly-lowland karst terrain}

In this type of the terrain, the material for the generation of the same indicators of mine presence as in the land type of the terrain is significantly different, but the approach in this part of the analysis is the same.

\subsubsection{Bunker, embankment and dry stone wall}

All above mentioned objects are made of the material (stone, concrete) with similar radiometric characteristics, and the very often appear together in this type of the terrain. The analysis has been performed on the basis of the images made by means of sensors in the following cameras: DuncanTech MS 4100 (RGB infrared image - IR, R and G; $0.4-0.9 \mu \mathrm{m} ; 1920 \times 1080$ pixels), Canon EOS 5D, Nikon D90 (RGB image; 4288x2848 pixels), RMK (RGB infrared image - IR, R and G; $0.4-0.9$ $\mu \mathrm{m}$; scanned with the size of pixels $0.03 \mathrm{~m}$ ), multispectral scanner DAEDALUS (11 channels; 0.4 - $14 \mu \mathrm{m}$; resolution 1 m) and on WorldView2 satellite (panchromatic orthorectified; resolution $0.5 \mathrm{~m}$ ).

The objects from the Figure 4, concrete bunker 1 and 2, embankments, trench 3 and the patterns of the four types of the surrounding area have been segmented and formed on the images of the sensors MS 4100, Nikon D90 and on the WorldView2 panchromatic satellite image (PAN), according to the parameters 40_7-3-7-3. All levels (images) have been geocoded before the segmentation on the basis of the satellite image and cut to the same size. The size of pixels of all created levels is $0.11 \mathrm{~m}$. Due to various resolutions, the level (channels) have been weighted before performing the segmentation, the largest weight (1.0) has been attributed to the RGB channels of the sensor Nikon D90, somewhat smaller (0.9) to the channels of the sensor MS 4100, and the smallest (0.7) to the satellite panchromatic image WorldView2.

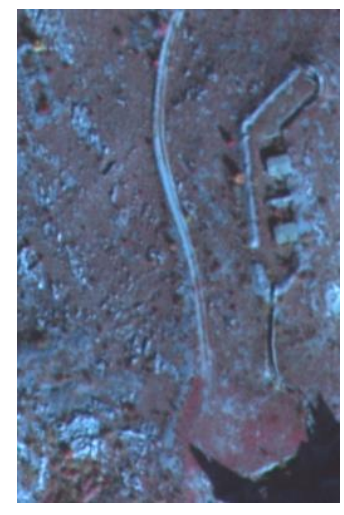

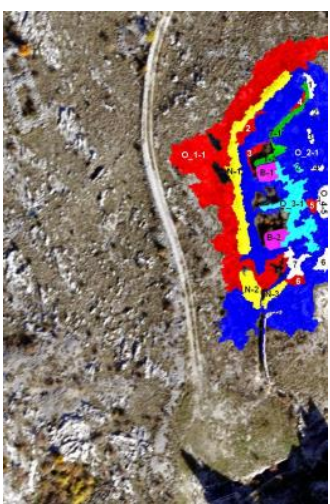

b
Figure 4. a) The cut part of the colour (RGB) infrared image (VNIR, MS 4100) of the determined position, and b) the presentation with two bunkers (pink), with embankment (yellow), with communication route between them (green) and the surrounding area (red, light and dark blue, and white) on the RGB image (Nikon D90) in the hilly-mountain type of the terrain (ITF, 2010).

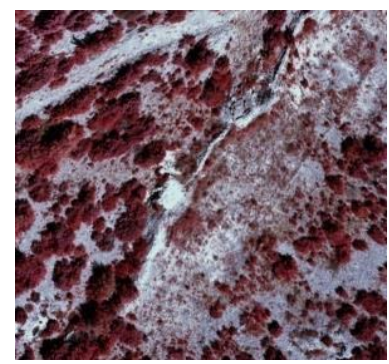

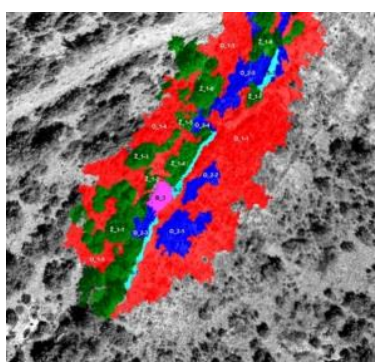

Figure 5. a) The cut part of the RGB infrared image (RMK) of the determined position and $b$ ) the presentation of the stone bunker 3 (pink), dry stone wall (light blue) and the pattern of 3 types of the surrounding area (red, dark blue and green) on the image of the green $(\mathrm{G})$ channel (RMK).

In the analysis of the stone bunker 3 and dry stone wall in the hilly-mountain type of the terrain (Figure 5a), the channels of RMK and DAEDALUS sensors have been used. Before the segmentation, the RMK RGB image has been geocoded on the basis of DAEDALUS images and all levels (images) cut to the same size with the size of the pixels of $0.03 \mathrm{~m}$ (the size that 
the RMK RGB image has been scanned with). The segmentation has been performed on the basis of the weighted channels, the RGB channels have been attributed the weight 1.0; and DAEDALUS channels the weight 0.7. After the segmentation on the basis of the parameters: 120_7-3-7-3, the smaller image objects have been joined into the meaningful objects: bunker 3, dry stone wall, the patterns of 3 types of surrounding area (Figure $5 b$ ).

\section{ANALYSIS OF THE FEATURES OF GENERAL INDICATOR SHAPES}

Radiometrical characteristics of the observed strong indicators of mine presence cannot provide the satisfactory discrimination of indicators as related to the surrounding area, but also the mutual one. For the purpose of better definition and discrimination of the indicators from the surrounding area, the object-based analyses of several types of features of general shapes of individual indicators types have been made (Krtalic, 2011).

Features of general shapes used in the analysis are the following (DEFINIENS, 2006):

- length/width (LW) - There are two ways to approximate the length/width ratio of an image object:

- The ratio length/width is identical to the ratio of the eigenvalues of the covariance matrix with the larger eigenvalue being the numerator of the fraction.

- The ratio length/width can also be approximated using the bounding box.

-asymmetry (ASY) - The lengthier an image object, the more asymmetric it is.

-density (DEN) - The density can be expressed by the area covered by the image object divided by its radius.

-shape index (SI) - Mathematically the shape index is the border length of the image object divided by four times the square root of its area A.

-border index (BI) - Similar to shape index, but border index uses a rectangular approximation instead of a square.

- compactness (COM) - This feature is similar to the border index, however instead of border based it is area based.

-roundness (ROU) - Formerly called: Diff. of enclosing/enclosed ellipse as the radius of the largest enclosed ellipse is subtracted from the radius of the smallest enclosing ellipse.

-elliptic fit (ELF) - The first step in the calculation of the elliptic fit is the creation of an ellipse with the same area as the considered object. In the calculation of the ellipse, the proportion of the length to the width of the Object is regarded. After this step, the area of the object outside the ellipse is compared with the area inside the ellipse that is not filled out with the object.

- rectangular fit (REF) - A first step in the calculation of the rectangular fit is the creation of a rectangle with the same area as the considered object. In the calculation of the rectangle, the proportion of the length to the width of the object in regarded. After this step, the area of the object outside the rectangle is compared with the area inside the rectangle, which is not filled out with the object.

- radius of smallest enclosing ellipse (RSE) - An ellipse with the same area as the object and based on the covariance matrix. This ellipse is then enlarged until it encloses the object in total. The ratio of the radius of this smallest enclosing ellipse to the radius of the original ellipse is returned for this feature.
- radius of largest enclosed ellipse (RLE) - An ellipse with the same area as the object and based on the covariance matrix. This ellipse is then scaled down until it's totally enclosed by the object. The ratio of the radius of this largest enclosed ellipse to the radius of the original ellipse is returned for this feature.

On the basis of these features, the subjective and factor analysis has been made. It has been done for the purpose of determining the features of general shapes on the basis of which strong indicators of mine presence could be better defined and discriminated among themselves. Based on these parameters, further research is planned aiming to determine the parameters for the monitored classification. The validity of this method will be tested in this way.

\subsection{Subjective analysis of feature value diagram of general shapes}

According to the value diagram of individual features of general shapes according to the individual strong indicators of mine presence, i.e. according to the features, it is possible to notice the similarities in their appearance and to presume the number of various clusters (groups) that the elements within these two groups can be divided into. All diagrams have been reviewed and the groups of features and indicators noticed having similar diagram shapes, i.e. similar characteristic related to the individual feature, i.e. indicator (Krtalic, 2011).

\subsubsection{Diagram of value analysis according to the features} The features of general shapes are a group smaller in number, but their diagrams, i.e. the values according to strong indicators of mine presence, differ much more, and they are therefore divided into five clusters ( Table 2).

\begin{tabular}{|c|c|c|c|c|}
\hline 1. cluster & 2. cluster & 3. cluster & 4. cluster & 5. cluster \\
\hline $\begin{array}{c}\text { BI, COM, } \\
\text { L/W, ROU, } \\
\text { SI }\end{array}$ & $\begin{array}{c}\text { DEN, } \\
\text { ELF, REF }\end{array}$ & ASY & RSE & RLE \\
\hline
\end{tabular}

Table 2. Subjective division of the features of general shapes according to features in five clusters.

The first cluster contains strong indicators of mine presence having the length essentially bigger than the width (embankments in the form of linear object, trenches, battlements). According to this feature of the general shape, small more compact objects (bunkers, shelters, fords) having essentially smaller values than the long objects can be discriminated. More winding objects have smaller values in this cluster because of big lengths and small area. Hence, the features are not in proportion to the length, except for length/width (L/W) (that depends on the length of the indicator, but not as much as asymmetry (ASY)), as it is the case with the asymmetry in the third cluster. By means of this feature, it is also possible to discriminate smaller and shorter objects (bunkers, shelters, fords), and the values for long objects are much more uniform (dry stone wall, embankment in the form of linear object, trench, battlement). By means of this parameter, unlike those from the first cluster, another long object can be discriminated: dry stone wall. The features of general shapes from the second cluster are some kind of a negative of the features from the first cluster. Namely, smaller and more compact indicators (bunkers, embankments in the 
form of a surface, shelter) have essentially larger values than the long objects.

The diagrams of the remaining two features (RSE, RLE) have more unique structure, and, since they cannot be classified into neither of three mentioned cluster, they each make a cluster for themselves. The usage of the features of the fourth and fifth clusters is very disputable, and they are more significantly shown on long, linear objects (some trenches and battlements).

Based on the above mentioned, the subjective analysis of the diagram of feature values according to the strong indicators of mine presence shows that there are five clusters with various classification of values (Table 2), however, if the clusters having no perspective of indicator resolution are neglected (semi axes of the smallest enclosing (RSE) and the largest enclosing ellipse), or if they are listed into one of the three remaining clusters, three more meaningful clusters are formed. These three clusters correspond to the division of strong indicators of mine presence into surface and linear objects. The first and the second clusters distinguish mostly less compact objects from the long objects, while the third cluster does the same, but its values are more uniform (less distinct) than the features from the first two clusters.

\subsubsection{Diagram of feature values according to indicators}

In the case of diagrams according to strong indicators of mine presence (there were 17 characteristic indicators selected for this analysis), it was much easier to classify the indicators according to features into three meaningful clusters ( Table 3).

\begin{tabular}{|c|c|c|}
\hline 1. cluster & 2. cluster & 3. cluster \\
\hline Bunker_K1 & Embankment_K1 & Trench_B7 \\
\hline Bunker_K2 & Embankment_K4 & Trench_B10 \\
\hline Bunker_K3 & Trench_K11 & Battlement_B2 \\
\hline & Shelter_B1 & Battlement_B4 \\
\hline
\end{tabular}

Table 3. Subjective classification of features of general shapes according to strong indicators of mine presence in three clusters.

This classification into clusters also makes it possible to notice the division into smaller, shorter and more regular objects (the first cluster), longer (linear) objects (the second cluster) and the third cluster that contains less distinctive representatives of its type. The trenches are (as it has been previously described) mostly long winding objects, but one can find in the nature also smaller trenches or sections that are straight (of regular shape), so they do not have the features of a classical continuous trench and are withdrawn from one of the first two defined clusters. Hence, the classification of feature values according to the indicators is more uniform as related to the form, but it differs related to the intensity of an individual feature. As an example, one can take the feature values length/width (L/W) and asymmetry (ASY) for bunkers and bridges. The material they are made of can be, and mostly is similar (concrete, stone), but they differ essentially according to the values of these two features.

\subsection{Factor analysis of the values of general shape features}

The confirmative factor analysis according to the method of main components has been performed in two ways. In the first case, the manifested variables have been analysed according to the features of general forms (the columns of the Table 2), and in the second case according to the strong indicators of mine presence (the rows of the Table 3). The aim of the factor analysis: to determine the degree of (non)connection, i.e. of mutual relations of IMPs according to the features of general shapes and the features according to the strong indicators of mine presence (Krtalic, 2011).

\subsubsection{Factor analysis according to features}

After performing the factor analysis according to five parameters and varimax rotation, the factor loadings presented in the Table 4 have been obtained. It can be seen (Table $4 \mathrm{a}$, principal components) that over $95 \%$ of the variances from the set has entered the analysis, hence the criterion of the variance significance level has been met (Pavlic, 1984), although it is not the presumption for the factor analysis intended for testing of various parameters that can be used to discriminate the strong indicators of mine presence.

The first factor has highly negative factor loading for the features of the general shape: density (DEN), semi axes of the smallest enclosing (RSE) and the largest enclosing ellipse (RLE), and we can call it the factor of lowest density of strong indicators of mine presence, because the values of all three features mark the degree of indicator density. The second factor (that the negative loading of the feature density DEN is confused with) has large positive loading with the features characterizing the length of the object, thus, we can call it the factor of indicator length. The next two factors have high independent loading because they have essentially different classification of values than the others, and they are called conditional factors. The fifth factor has relatively high positive loadings on the features of border index (BI), compactness $(\mathrm{COM})$, roundness (ROU) and shape index (SI) that discriminate less compact objects, thus we call it the factor of compactness.

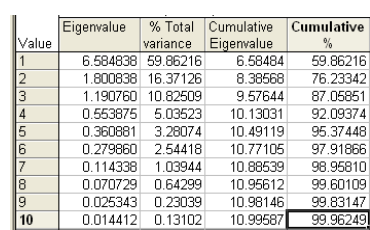

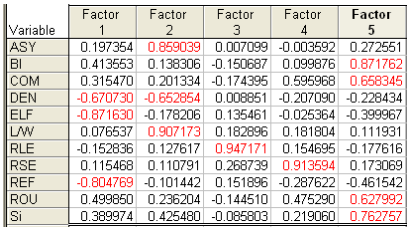

b
Table 4. a) The values of the principal components and b) factor loadings after varimax rotation of three factors.

The first assumption that we started the factor analysis with to analyse the possibility of dividing the features into five clusters has thus been confirmed, with the difference that the feature length/width (L/W) has "inclined" toward the feature asymmetry (ASY) (Figure 6).

Since the factor analysis is used for the determination of less latent variables that sum up in adequate way the whole set of manifested variables, and in this case we have the factors with only one larger loading and the presumption that the observed set can be described with smaller number of factors, the factor 
analysis has been repeated, but this time with three factors (Figure 7a), in order to place two features from the third and the fourth factor into a wider set of features.

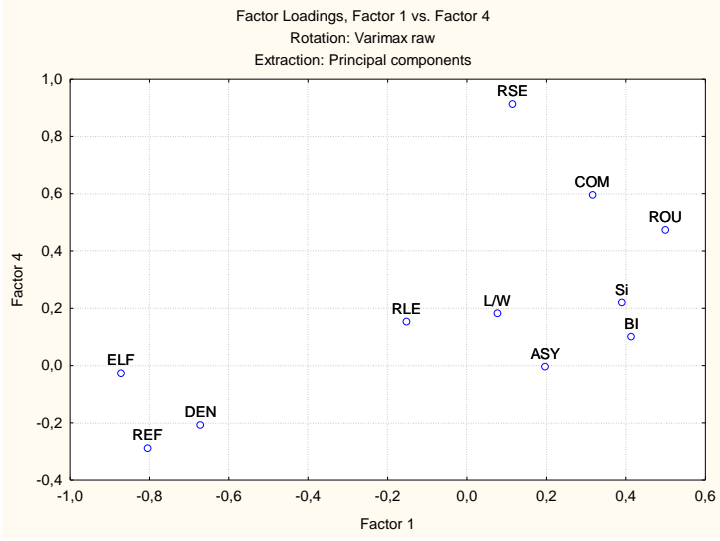

Figure 6. 2D presentation of the values of factor loadings of the first (X axis) and the fourth factor ( $\mathrm{Y}$ axis) from the Table $4 \mathrm{~b}$.

The results of this analysis is the classification of features into three factors, namely in the following way. The first factor has large positive loadings for the features border index (BI), compactness $(\mathrm{COM})$, roundness (ROU) and the shape index (SI) (Table 5-9a), and it is called compactness factor, and large negative loadings for the features elliptic fit (ELF) and rectangular fit (REF) that also show the same, the parameters of compactness coincide, and the other values are inversely proportional. The second factor is the length factor with large positive loadings on the features asymmetry (ASY) and length/width $(\mathrm{L} / \mathrm{W})$, and negative loading on the feature density DEN (Figure 7b), which indicates low density of long and irregular IMPs (trenches, battlements). The third factor is called corrective factor (without noticeable characteristic of discrimination, presuming that some linear objects are still distinctive due to these features) encompassing the nonrepresentative objects distinguished in the first two factors. Thus, two presumptions are confirmed. The first is the classification of the indicators of mine presence in surface (less compact objects: trench, surface embankment, fords) and linear objects, and the second presumption is related to three clusters in which the features of general indicator shape can be placed.

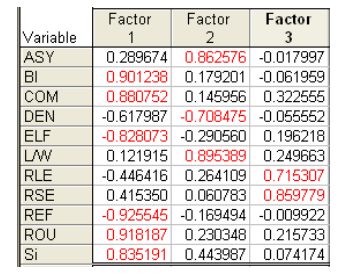

a

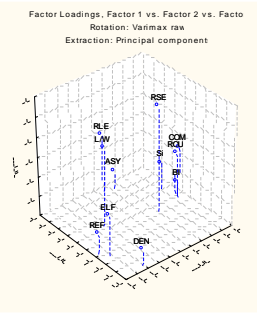

$\mathrm{b}$
Figure 7. a) Factor loadings after varimax rotation of three factors. b) 3D presentation of the values of factor loadings of the first ( $\mathrm{X}$ axis), the second ( $\mathrm{Y}$ axis) and the third factor ( $\mathrm{Z}$ axis) from a.

\subsubsection{Factor analysis according to indicators}

In this case, the factor analysis has been made according to three parameters and, after varimax transformation, the factor loadings have been obtained and presented in the Table 5b. It can also be seen (Table 5a) that the first three main components containing over $97 \%$ of the total variance of the set have entered into the analysis (Krtalic, 2011).

The first factor has highly positive factor loading for strong indicators of mine presence whose length is more dominant as related to its width (dry stone wall, embankment, trench, battlement) and it can be called length factor. The second factor has large loadings for compact, shorter objects of more regular shape (bunker, shelter for artillery or tank), and this factor is called compactness factor. In the third factor, the loadings are relatively high for the linear indicators and this factor is called corrective factor which encompasses all nondistinctive cases from the previously described factors (trench, battlement, Figure 8).

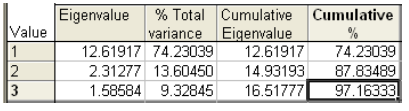

a

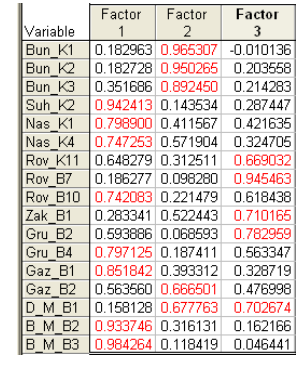

b
Table 5. a) The value of the principal components and b) factor loadings after varimax rotation of three factors.

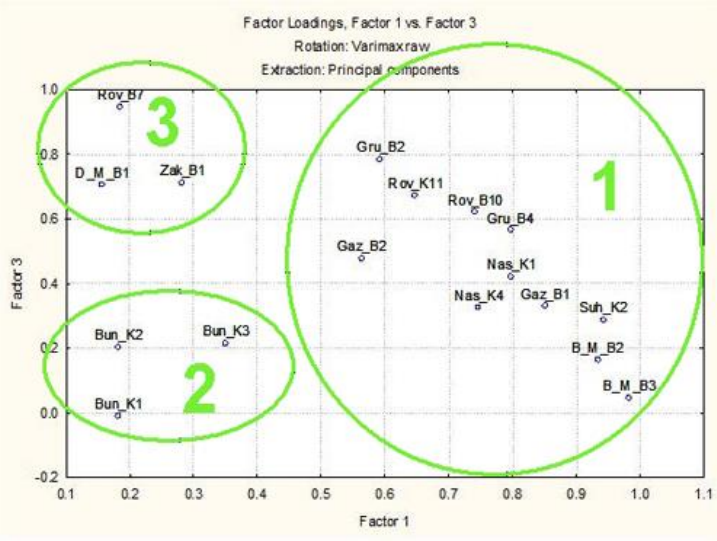

Figure 8. 2D presentation of the values of factor loadings of the first ( $\mathrm{X}$ axis) and the third factor ( $\mathrm{Y}$ axis) from the Table $5 \mathrm{~b}$.

\section{CONCLUSION}

The indicators of mine presence are of great importance and essential for the whole processing within the system supporting the decision making in undetermined conditions. It is mainly related to strong indicators of mine presence that are interactively separated by the interpreters of the mine scene, because they can be hardly separated by means of automatic processing on the basis of their spectral and radiometric characteristics. In order to define the indicators of mine presence more precisely, this paper present the classification of strong indicators of mine presence (trench, shelter, bunker) in two groups: linear and surface, and the indicators are defined 
by means of the features of general shapes; (asymmetry, border index, compactness, density, elliptic fit, length/width, radius of smallest enclosing ellipse, radius of largest enclosed ellipse, rectangular fit, roundness). The performed analyses (subjective, factor analysis) show that the tested strong indicators of mine presence can be distinguished mutually and related to the surrounding area by means of defined values of general shape features. This work introduces the interactive distinction of strong indicators of mine presence by means of image segmentation and the features of individual segments (general shapes, spectral, radiometric). Subjective distinction of the indicators of mine scene on the basis of basic images and true data related to the terrain (object image from the ground) is replaced by interactive joining of the segments having the same features that finally make a distinctive indicator of mine presence. Such defining and interactive distinction of strong indicators of mine presence is a significant step further as related to so far performed analyses of strong indicators of mine presence and one of important scientific contributions of this work.

Further research devoted to this topic should detect the influence of the characteristics (radiometric and geometric) of individual images on the quality of segmentation. It is planned to apply the method for other objects of interest as well that depend more on the accuracy of segmentation, which was not the case with IMPs. Furthermore, the research is further continued also on the establishment of rule sets for the performance of automatic processing (feature extraction, object-oriented classification) intended for the separation of the indicators of mine presence according to their features of general shapes.

\section{ACKNOWLEDGEMENTS}

This paper is the result of the research that started within the scope of the projects Deployment of the Decision Support System for Mine Suspected Area Reduction (ITF, 2008) and Deployment of the Decision Support System for Mine Suspected Area Reduction in Bosnia and Herzegovina (ITF, 2010) funded by the US State department through International Trust Fund for Demining and Mine Victims Assistance (ITF) Ig, Slovenia and was continued within the scope of the project Toolbox Implementation for Removal of Anti-personnel Mines, Submunitions and UXO (TIRAMISU, 2012-2015) funded by the European Union's Seventh Framework Programme under grant agreement no 284747 .

\section{REFERENCES}

ARC (2004): ARC Final Public Report, Project funded by the European Community under the "Information Society Technology" Programme (1998-2002)

Acheroy, M.; Yvinec, Y. (2008): Mine-suspecte Area Reduction Using Aerial and Satellite Images, Humanitarian Demining: Innovative Solutions and the Challenges of Technology, Book edited by: Maki K. Habib, ISBN 978-3902613-11-0, pp. 392, February, I-Tech Education and Publishing, Vienna, Austria

Bajic, M.; Gold, H.; Fiedler, T.; Gajski, D. (2008): Development of the concept from 1998 and realisation in 2007 - 2008 of the system for the airborne multisensor recconnaissance and surveillance in the crisis situations and the protection of the environment, in Proceedings of Disaster Management and Emergency Response in the Mediterranean Region, Oluic (ed.), First EARSeL Conference, ISBN 978-953154-876-2, Zadar, Croatia, September 2008, str. 401-410.

Bajić, M. (2010). The advanced intelligence decision support system for the assessment of mine suspected areas, Journal of ERW and Mine Action, James Madison University, Issue 14.3, Fall 2010, pp. 69-75,

DEFINIENS (2006): Definiens Professional 5, Reference Book, Definiens AG, München, Germany

ITF (2008): Deployment of the Decision Support System for Mine Suspected Area Reduction, International Trust Fund for Demining and Mine Victims Assistance (ITF) Ig, Slovenia

ITF (2010): Deployment of the Decision Support System for Mine Suspected Area Reduction in Bosnia and Herzegovina, International Trust Fund for Demining

Krtalić, A. (2011): Sustav za potporu odlučivanju u uvjetima neodređenosti u protuminskom djelovanju utemeljen metodama daljinskih istraživanja, Doctoral thesis, Faculty of Geodesy University of Zagreb, Zagreb (in croatian).

Krtalic, A. (2012): Thematic Maps for Humanitarian Demining I Tematske karte u humanitarnom razminiranju, Cartography and Geoinformation: Scientific and Professional Information Journal of the Croatian Cartographic Society 11, 17; 26-41.

Krtalic A.; Bajic M. (2013). Upgrade of the Advanced Intelligence Decision Support System for Mine Action in the TIRAMISU project, 11th IARP Workshop HUDEM 2013, 25th April 2013, Sibenik, Croatia, book of papers, pp.83-86

Pavlic, I. (1984): Statistička teorija i primjena, Tehnička knjiga, Zagreb (in croatian)

SMART D5.1 (2004): Scientific report of processing algorithms, SMART, EC IST-2000-25044, SMART-D5.1_v1

van Genderen, J.; Maathuis, B. (1999): Pilot Project for Airborne Mine-field Detection in Mozambique, EC-DG8, UK, Netherlands, Luxembourg, Sweden, United Kingdom, Portugal, BelgiumGermany, Norway, Angola project

Vanhuysse, S. (2002): Mission Report (fieldwork in Croatia), SMART, EC IST-2000-25044

Navulur, K. (2006): Multispectral Image Analysis Using the Object-Oriented Paradigm, CRC Press, Taylor \& Francis Group, Boca Raton, U.S.

URL1: StatSoft, http://www.statsoft.com (15/4/2016)

URL2: Definiens, http://www.definiens.com (15/4/2016)

URL3: TIRAMISU project, http://www.fp7-tiramisu.eu/projectoverview $(15 / 4 / 2016)$ 